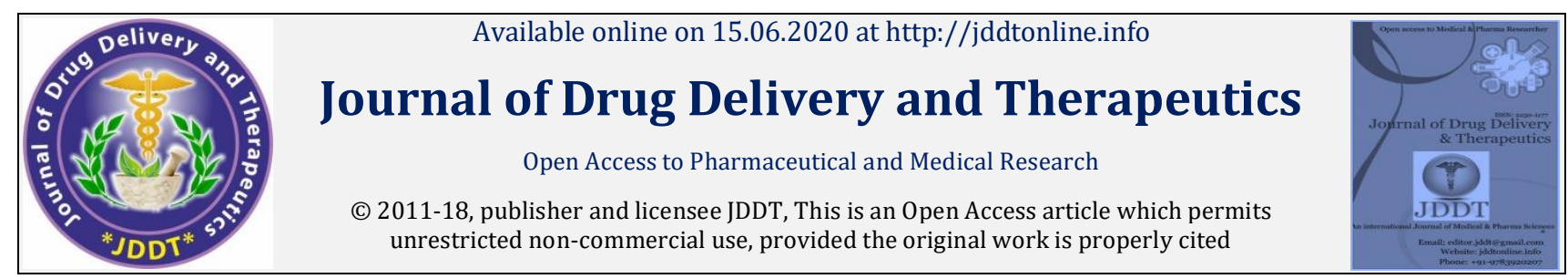

Open $\odot$ Access

Case Report

\title{
A Case Report on Viral Meningoencephalitis with Radiculoneuropathy
}

\author{
Ali Salman Mir*, Faizan Abdullah Md, Baleeqhuddin Mohammed \\ Deccan school of pharmacy, Dar-Us-Salam, Aghapura, near Nampally, Hyderabad, Telangana 500001
}

\begin{abstract}
Meningoencephalitis is an inflammation of brain and its surrounding membranes. It can be caused by bacteria, viruses, fungi, protozoa and by various other etiology. Radiculoneuropathy, also commonly referred to as pinched nerve, refers to a set of conditions in which one or more nerves are affected. The classic triad of fever, stiff neck and altered sensorium are seen in $<50 \%$ of all patients with meningitis. Clinical presentation of viral encephalitis includes a prodrome of fever, headache, myalgia, and mild respiratory infection. Altered mentation, focal neurological deficits and seizures usually follow. Patient was brought to emergency department of hospital. He provided with empirical antibiotic therapy and supportive care. Laboratory investigation like CSF analysis, liver function test, renal function test. Blood test like complete blood picture, dengue Ag. Microbiological test was also carried out. Radiological imaging like MRI of Brain, MRI of spine, Chest X-Ray. Upon clinical diagnosis patient was provided with antibiotic, antiviral and supportive care. Patient was also provided with mechanical ventilator. He was treated for 14 days.
\end{abstract}

Keywords: meningoencephalitis, CSF analysis, classical triad.

Article Info: Received 28 March 2020; Review Completed 22 May 2020; Accepted 29 May 2020; Available online 15 June 2020

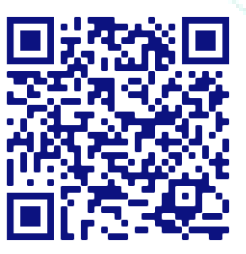

Cite this article as:

Ali SM, Faizan AM, Baleeqhuddin M, A Case Report on Viral Meningoencephalitis with Radiculoneuropathy, Journal of Drug Delivery and Therapeutics. 2020; 10(3-s):231-233 http://dx.doi.org/10.22270/jddt.v10i3-s.4168

*Address for Correspondence:

Ali Salman Mir, Deccan school of pharmacy, Dar-Us-Salam, Aghapura, near Nampally, Hyderabad, Telangana 500001

\section{INTRODUCTION}

We present the case of a 41-year-old male with a history of recent Kerala travel who presented with characteristic clinical features of meningoencephalitis. Cerebrospinal fluid (CSF) analysis shows abnormality. Nerve conduction study (NCS) showed radiculoneuropathy. Meningoencephalitis is an inflammation of brain and its surrounding membranes. It can be caused by bacteria, viruses, fungi, protozoa and by various other etiology. This is the largest study to date evaluating the epidemiology, management, and outcomes of adults with meningitis or encephalitis in the United States. The median age of the patients was 43 years; half of them were nonwhite and $16.4 \%$ had a significant number of comorbidities, with $4.2 \%$ having HIV coinfection. As seen in other studies, patients with meningitis and encephalitis have high rates of hospitalization and empiric antimicrobial use $1,2,3$

The majority of the patients were evaluated and admitted through the emergency departments, but up to $22.5 \%$ of lumbar punctures were done as an inpatient. This could potentially be explained due to a trend toward interventional radiologists performing the lumbar punctures instead of the treating physicians 4
Etiology for meningoencephalitis is by bacteria (listeria monocytogenes, several species of Rickettsia, Mycoplasma pneumoniae, mycobacterium tuberculosis, borrelia and leptospirosis), by viruses (west Nile virus, measles virus, Epstein-Barr virus, Varicella-zoster virus, Enterovirus, Herpes simplex virus type1, Herpes simplex virus type2, Rabies virus, HIV). Other causes include autoimmune, protozoal and fungal.

Clinical presentation of viral encephalitis includes a prodrome of fever, headache, myalgia, and mild respiratory infection. Altered mentation, focal neurological deficits and seizures usually follow. 5 The classic triad of fever, stiff neck and altered sensorium are seen in $<50 \%$ of all patients with meningitis. Ninety-five percent of patients will, however, have two out of the four symptoms of headache, fever, neck stiffness and altered consciousness 6

Specific neurological findings include mental status, Glasgow Coma Score, state (alert, disoriented/awake, stuporous, coma/decorticate or coma/decerebrate), the presence of photophobia, aphasia, abnormalities of cranial nerves (ophthalmoplegia, facial paralysis, ptosis), deep tendon reflexes, motor (hemiparesis) and sensory and cerebellar (ataxia) defects. Findings should be described as to whether they are intact or abnormal, their symmetry and focality ${ }^{7}$ 
Neuroimaging studies such as MRI and CT scan. If there is a increased intracranial pressure with focal signs, this should be undertaken before a lumbar puncture in order to rule out other lesion such as abscess, subarachnoid hemorrhage, tumors and stroke and to localize lesions.

The usual CSF findings are elevated protein, normal glucose levels and a pleocytosis ( 7 cells $/ \mu \mathrm{L}$ or more) with a predominance of mononuclear cells. An exception is Acute hemorrhagic encephalomyelitis in which polymorphonuclear cells predominate. Also, especially early in the course of illness and hospitalization, CSF may be normal or show hypoglycorrachia and a predominance of polymorphonuclear cells. Approximately $3 \%$ to $5 \%$ of patients with severe viral infections of the CNS have no CSF pleocytosis on initial CSF examination. While pleocytosis should not be attributed to status epilepticus until all other causes have been ruled out, a white blood cell count of more than $6 \times 10^{6} / \mathrm{L}$ or one or more polymorphonuclear leukocytes have been found in $22 \%$ of these patients 8

Electroencephalography may further support the diagnosis by showing general findings compatible with encephalopathy. Of particular value in herpes simplex encephalitis is its characteristic periodic high voltage spike wave activity in the temporal regions and slow wave complexes (intervals of 2 to $3 \mathrm{~s}$ ). ${ }^{7}$

Determination of antiviral antibody synthesis in the CSF is usually not undertaken because it has not been considered useful in the diagnosis of HSV infections 9, 10

Brain biopsy can be should be used in patients which do not respond to acyclovir and for whom there is a unknown etiology on neuroimaging studies and a deteriorating clinical condition.

Empirical therapy of antibiotics, antivirals and supportive care until investigation are carried out and final diagnosis is made.

\section{CASE REPORT: -}

A 41year old male patient who is chronic alcoholic and diabetic was brought to the emergency department of Owaisi hospital and research centrewith complaints of fever and chills, drowsiness since 2 days on examination patient was sleepy, on admission his vitals were temp:- $99.6 \mathrm{~F}, \mathrm{P} / \mathrm{R}:-$ 99bpm, B.P:- 100/80mmhg, , neck stiffness:- +ve ,GCS:E3V3M6:- 12/1.5, Kernig's sign: - +ve.

The patient had travel history to Kerala where he developed fever $\mathrm{H} / \mathrm{O}$ cough in the last 10 days

Patient has been diabetic for 2 days and alcoholic for 3 years.

Investigation: -

Blood Test

Lymphocytes: - 52\% (Normal value: - 20\%-40\%)

Dengue ns1 Ag- negative

Microbiology

Cytopathology; - predominantly lymphoid cell. No atypical cells.

\section{CSF Analysis}

Glucose: - $122 \mathrm{mg} / \mathrm{dL}$ (Normal value: - 45mg/dL$80 \mathrm{mg} / \mathrm{mg} / \mathrm{dL}$ )

Protein: - $96 \mathrm{mg} / \mathrm{dl}$ (Normal value: -15mg/dL-45mg/dL)

ADA level: - $5.9 \mathrm{U} / \mathrm{L}$ (Normal value: up to $5 \mathrm{U} / \mathrm{L}$ )
Nerve Conduction Study

Demyelinating sensory motor poly radiculoneuropathy.

Radiology

MRI of Lumbar Spine; - on day 3

Changes of degeneration.

Mild disc bulge at L4-L5 level causing indentation over thecal sac.

Mild disc protrusion at L5-S1 level causing indentation over thecal sac, mild compromise of bilateral neural recesses.

MRI of Brain: -

Subtle restricted diffusion, T2 FLAIR subtle Hyperintense areas in left side of pons and left side of mid braininflammation.

Small T2 FLAIR Hyperintense area in bilateral periventricular/subcortical white matter- inflammation.

Chest X-ray was normal.

Differential Diagnosis: -

Physical examination like classic traid of fever, neck stiffness and altered sensorium is seen and kernig's sign is seen. Investigation finding like CSF analysis, cytopathology. Based on the physical examination and investigation finding, it has been diagnosed as meningoencephalitis.

Treatment: -

Upon admission patient was given empirical antibiotic therapy of Intravenous ceftriaxone 2gm twice a day and after clinical diagnosis patient was given intravenous acyclovir $500 \mathrm{gm}$ thrice a day along with Intravenous ceftriaxone $2 \mathrm{gm}$ twice a day and tablet paracetamol 500mg thrice a day. As the patient was severely ill, he was needed mechanical ventilator. The patient was kept on mechanical ventilator until day 4. Upon diagnosis of radiculoneuropathy, he was provided with appropriate physiotherapy. The patient was isolated to prevent transmission with proper precautions.

Outcome and Follow up: -

The patient was on mechanical ventilator till day 4 and remained haemodynamically stable. Patient was symptomatically improved during her 14-day admission. Patient on time of discharge was feeling well.

\section{DISCUSSION}

Meningoencephalitis is medical emergency which require early medical attention. Meningoencephalitis is not quite often seen. Many patients can be seen with either meningitis or encephalitis.

Upon physical examination, investigation finding, imaging finding. Patient was diagnosed with viral meningoencephalitis along with radiculoneuropathy. Patient had to provide with mechanical ventilator upon admission. Patient was provided with empirical antibiotic therapy, antiviral and supportive care. The patient was isolated to prevent transmission with proper precautions.

\section{REFERENCES}

1. Khoury NT, Hossain MM, Wootton SH, Salazar L, Hasbun R. Meningitis with a negative cerebrospinal fluid Gram stain in adults: risk classification for an adverse clinical outcome. Mayo Clinic Proc 2012; 87:1181-8 
2. Hasbun R, Bijlsma M, Brouwer MC, et al. Risk score for identifying adults with CSF pleocytosis and negative CSF Gram stain at low risk for an urgent treatable cause. J Infect 2013; 67:102-10.

3. George BP, Schneider EB, Venkatesan A. Encephalitis hospitalization rates and inpatient mortality in the United States, 2000-2010. PLoS One 2014; 9:e104169

4. Kroll H, Duszak R Jr, Nsiah E, Hughes DR, Sumer S, Wintermark M. Trends in lumbar puncture over 2 decades: a dramatic shift to radiology. AJR Am J Roentgenol 2015; 204:15-9.

5. Sapra H, Singhal V. Managing Meningoencephalitis in Indian ICU. Indian J Crit Care Med. 2019; 23(Suppl 2):S124-S128.

6. van de Beek D,, de Gans J,, Spanjaard L,, Weisfelt M,, Reitsma JB, Vermeulen M. Clinical features and prognostic factors in adults with bacterial meningitis. N Engl J Med. 2004; 351((18):):1849--1859.

7. Ford-Jones, E L et al. "Acute childhood encephalitis and meningoencephalitis: Diagnosis and management." Paediatrics \& child health vol 3

8. Barry E, Hauser WA. Pleocytosis after status epilepticus. Arch Neurol. 1994; 51:190-3

9. Jeffrey JKM, Read SJ, Peto TEA, et al. Diagnosis of viral infections of the central nervous system: clinical interpretation of PCR results. Lancet. 1997; 349:313-7

10. Lakeman FD, Whitley RJ, the NIACA Study Group Diagnosis of herpes simplex encephalitits: application of polymerase chain reaction to cerebrospinal fluid from brain-biopsied patients and correlation with disease. J Infect Dis. 1995; 171:857-63 